\title{
La Plume androgyne d'Angeline Solange Bonono : du féminin à la masculinisation de l'écriture
}

\author{
Raymond Mbassi Atéba \\ Université de Yaoundé I
}

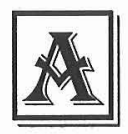

trop souvent écouter les voix féminines francophones diversement célébrées à travers le fait littéraire, malgré leurs positions parfois tranchées sur certains problèmes de société, on oublie de réserver une oreille attentive à celles qui, même arrivées récemment dans la création littéraire, renouvellent le débat sur les genres et l'orientent peut-être vers d'autres perspectives. Et pourtant, la recrudescence de nouveaux talents féminins ayant la capacité d'influencer le champ littéraire dans son interaction avec le champ socio-politique (Pierre Bourdieu, 1992; Edmond Cros, 1998) amène à considérer - qu'on le veuille ou non - les nouvelles générations d'écrivaines comme des voix en quête d'une certaine légitimité. La poétesse camerounaise Angeline Solange Bonono est certainement l'une de ces " voix de l'ombre " habitée, elle aussi, par les peurs, les angoisses et les obsessions chroniques de la littérature féminine francophone de première heure. Mais où ses devancières s'illustrent par un féminisme différentialiste, nimbé de radicalisme et de moralisme, mettant un point d'honneur sur l'égalité entre les hommes et les femmes, l'auteure de Soif Azur privilégie le féminisme universaliste et libéral, où la féminité et la masculinité se neutralisent dans l'humain.

Sa parole scripturale révolutionne une mesure verbale adossée sur les sacro-saints classiques éthologique, psychologique et esthético-thématique collés à chaque genre. Elle opère une démesure de la mesure en déplaçant la féminité vers la masculinité dans un jeu de substitution, de fluctuation et d'équivalence, le but inavoué étant de parâtre, de sentir, de dire et de faire selon une condition masculine au détriment d'une condition féminine. Cette attitude s'inspire d'un intertexte social, qu'explicite Selim Yahi (1995: 163-164) : 
Le désir de se hisser socialement les pousse à adopter un certain nombre de comportements typiques dans le choix de leurs vêtements et de leur coupe de cheveux comme dans leurs prouesses intellectuelles et physiques. Malgré leur attitude délibérée, ces femmes ne semblent pas pour autant revendiquer le statut masculin même si certaines d'entre elles effacent dans leur apparence physique une grande partie de leurs caractéristiques féminines.

Effacer le féminin, pour se fondre dans le masculin : telle est l'impasse dans laquelle s'inscrivent certaines figures féminines aujourd'hui. Cette assimilation à la condition masculine inonde le champ socio-politique des scénarios et d'exemples parfois ubuesques : dans son rapport avec le pouvoir, la femme brille par une masculinisation voire une surmasculinisation qui rappelle la redoutable Infante de Navarre, dans La Reine morte de Henry de Montherlant. À la thématique féminine du pardon, de la délicatesse, de la pudeur se substitue la thématique masculine de la puissance, de la guerre, de l'indélicatesse, de l'obscénité, etc. Ce baroque postmoderne - toute époque classique est suivie d'une époque baroque investit la parole scripturale d'Angeline Solange Bonono à travers une énonciation spéculaire ambiguë, la volonté de puissance du féminin, le renoncement à la préciosité, l'inversion du désir. Cependant, la perspective universaliste du féminisme de cette poétesse valorise l'humain dans une discordante harmonie entre le féminin et le masculin.

\section{De la spécularité féminine à l'altérité masculine}

L'écrivain allemand Jean Wolfgang Goethe (1749-1832) propose certainement l'une des meilleures illustrations du narcissisme féminin, dans Faust, lorsqu'il fait dire à Marthe, pour rassurer la jeune Marguerite au sujet des bijoux offerts par Méphistophélès, (Jean Wolfgang Goethe, 1964: 117) :

Viens souvent me trouver, et tu essaieras ici en secret ces parures, tu pourras te promener une heure devant le miroir : nous y trouverons toujours du plaisir.

Cette promenade devant le miroir pour admirer et adorer sa propre image, on le sait, rappelle la théorie des reflets chère à Gérard Genette (1976 : 21). Selon lui, en effet, 
Le reflet est un thème univoque : le reflet est un double, c'est-à-dire à la fois un autre et un même. Cette ambivalence joue dans la pensée baroque comme un inverseur de significations qui rend l'identité fantastique (je est un autre) et l'altérité rassurante.

On le voit d'entrée de jeu dans le poème « Passionaria " (A. S. Bonono, 2002 : 10). La poétesse substitue son identité sexuelle féminine à une identité masculine. Malgré l'origine féminine du poème, c'est plutôt un point de vue masculin que suivra le lecteur :

Je la veux pour moi tout seul

Je lui ferai un fonds moral

Un fonds mental pour évacuer

Ses promesses cyclothymiques.

Le "Je " de l'écriture devient un moi polyvalent qui renvoie à une instance énonciative masculine. La parole scripturale de la poétesse ressemble ainsi à un miroir identitaire où l'altérité masculine est plus que spéculaire. Elle suppose l'effacement du féminin et l'affirmation, plus tard, d'une certaine virilité. On l'observe dans le poème "Alors " (A. S. Bonono, 2002:29) :

Je te remplirai de sensations nouvelles Je te foudroierai de tant de décharges brûlantes

Que tu voudrais mourir pour moi

Oui mourir pour une pauvre Quasimodo

Qui tiendra son Esméralda.

Il y a une double inversion de rôles dévolus au féminin : l'énergie sexuelle reconnue à l'homme - " décharges brûlantes " - est transférée à la femme; Quasimodo - homme - qui meurt pour Esméralda - femme -, dans le récit hugolien Notre Dame de Paris s'oppose à Quasimodo - femme - pour laquelle Esméralda - homme - meurt, dans Soif Azur. Au bout du compte, on est dans une ambiguïté qui, au-delà des travestissements descriptifs et énonciatifs, annule la question des genres à travers la trace d'une voix intime de la poétesse qui se décentre vers une altérité masculine. Mais pour mieux incarner cette masculinité, elle va aspirer à la toute puissance. 


\section{De la volonté de puissance à la parole virile}

Certains poèmes, à l'instar de "Impossible autrement " (A. S. Bonono, 2002 : 20), marquent la virilisation de la parole à travers un discours injonctif de la poétesse adressé à l'être aimé :

\section{Tu seras mon otage \\ Mon bel otage consentant \\ Toi le seul qui me fais craquer \\ Impossible autrement.}

Cependant, on dénote en filigrane l'autorité qui recouvre toute affirmation d'un pouvoir contraignant. Évidemment, le discours du sujet dominant brille par son agressivité et son impertinence, comme le précise Friedrich Nietzsche (1993 : 1110), commentant le sentiment de puissance chez Napoléon :

Napoléon enrageait de parler mal et ne s'en faisait pas accroire sur ce point : mais son désir de dominer, qui ne méprisait aucune occasion de se manifester et qui était plus subtil que son esprit subtil, l'amena à parler encore plus mal qu'il ne le pouvait.

En déplaçant la parole du champ socio-politique du début du $\mathrm{XIX}^{\mathrm{c}}$ siècle, où s'exerce la puissance de Napoléon, à Soif Azur, où la poétesse se masculinise à travers un processus d'impanation, où le féminin coexiste verbalement avec le masculin, on peut rattacher à la virilité un discours dénué de purisme et de préciosité qui rappelle certaines attitudes caractéristiques du masculin par opposition à celles qui relèvent de la thématique féminine. Le poème "Dérive » (A. S. Bonono, 2002 : 40), si on n'abuse pas, est l'un des rares où rejaillit un dernier sursaut de féminité à travers la pudeur de la poétesse et l'irruption de l'implicite :

Je le regarde

Elle me regarde

Elle est... comment dire?!

Indéfinissable

J'ai vraiment de la peine à salir

Ce que tu es... 
Ce répertoire bien pauvre des subtilités de la parole féminine, où l'implicite, les non-dits, les sous-entendus et autres énigmes meublent un discours pudique, où les silences sont tout aussi expressifs, ce répertoire de la bienséance et de la préciosité langagières des voix féminines d'antan, se décline sous la plume de cette poétesse camerounaise par une verve poétique volontairement pétrie d'indélicatesse. Le recueil Soif Azur en propose de nombreux exemples. Dans "Croupe au vent " (A. S. Bonono, $2002: 26$ ), on peut lire :

\author{
Croupe au vent \\ Fissure ouverte \\ Corps usé \\ Par trop d'amour-haine \\ Corps brûlé par trop de feu... \\ Corps vidé de son jus.
}

Cette dénonciation de la prostitution féminine est suivie par celle de la luxure masculine. Dans le poème " Pour que... " (A. S. Bonono, 2002 : 27), la poétesse écrit :

Des élections et des érections de Dieu

Des mariages des ovaires et des spermes

Des victoires sur des myomes séreux

Qui mettront fin aux hémorragies universelles

Pour que l'homme arrête de couler

Pour que l'homme arrête de dégouliner.

Dans la même lancée, elle s'oppose aux perversions sexuelles et aux sexualités parallèles, dans « Coup de gueule » (A. S. Bonono, 2002 : 30) :

Haro! à un monde immonde et incestueux

Où l'on coïte les foetus et les nourrissons

Dans les berceaux en sanglots. Halte aux uraniens!

Haro! à un monde merdeux et violeur

Où l'on coïte les bêtes devenues folles

Amantes des hommes.

On peut observer dans d'autres poèmes comme "Cerveau fêlé ", "Aria ", " Panacée " et " Chaos ", pour ne citer que ceux-là, la même hargne 
de dénoncer, à la manière des voix masculines, peut-être avec plus de pugnacité, les tragédies des temps présents. Dans le contexte d'une société patriarcale, habituée à considérer la femme comme le dépositaire de la morale et de la bienséance verbale, la parole scripturale de la poétesse Angeline Solange Bonono déplace les frontières de la mesure verbale féminine vers la démesure verbale masculine en hachurant la césure qui sépare les thématiques respectives des genres féminin et masculin et les champs lexico-sémantiques qui les accompagnent. Selon Edmond Cros (1998: 43),

En se reproduisant dans le sujet parlant, sous la forme d'une spécularité prétendument intériorisée, le Sujet, tout en se reproduisant comme tel, cache cette coïncidence avec lui-même, dans l'effet illusoire de l'autonomie du sujet parlant.

Cette autonomie du sujet dont il est question ici interfere aisément avec l'indélicatesse langagière de la poétesse qui n'est pas la marque stérile d'une écriture à la croisée des chemins mais l'affirmation ultime d'une identité sexuelle masculine acquise dans l'art. L'origine féminine de la parole poétique s'estompe pour céder la place à un discours viril, à la limite obscène. Cette parole scripturale de la poétesse qui se réinvente au masculin bouleverse l'orientation du désir entre les genres.

\section{L'inversion de l'orientation du désir}

La femme, de par son charme et sa sensualité, de par sa douceur, séduit, fascine, provoque et oriente le désir de l'homme. D'où les clichés tels que l'éternel féminin, où l'homme est détruit par la passion d'une femme, ellemême souvent prise pour un démon dans l'imaginaire judéo-chrétien plutôt favorable à l'inhibition du désir. Mais peut-on véritablement contenir le désir? Bernard Valette (1990: 41) observe à ce sujet :

Ce qui se trouve d'abord exalté, en même temps que condamné, est la force du désir : rien ni personne ne peut séparer Roméo et Juliette, Hernani et DoÀa Sol, plusiette, Hernani et DoÀa Sol, plus près de nous Bonnie et Clyde. Or ce désir est subversif. Il remet en cause l'ordre social et les valeurs morales.

En déchiffrant dans l'œuvre la nature spécifique du désir, comme le recommande Jean Starobinski (1964: 24), on peut tout de go observer que 
celui de la poétesse, dans Soif Azur, charrie des préoccupations émouvantes : elle remet en question d'abord le sens du désir. De la femme aimée, charmante et séduisante, provoquant le désir de l'homme, on aboutit sous sa plume à un homme charmant et séduisant, activant le désir de la femme. Il s'agit de l'image peu ordinaire de la femelle à la recherche du mâle, de la femelle conquérante, conquise par le mâle. On peut le voir dans " Pour que tu me reviennes" (A. S. Bonono, $2002: 27$ ) :
J'oublierai tes coucheries imbéciles à tous vents,
Je ne me vengerai pas de tes infidélités,
Pour que tu me reviennes,
Je te supplierai à genoux en baisant tes pieds,
Je ravalerai mes larmes écarlates, mes humeurs mes sangs,
Pour que tu me reviennes.

Dans la même optique, la poétesse réussit à transformer la portée du mythe de l'éternel féminin. De la déstructuration et la destruction de l'amant par la femme, on assiste à une destruction de l'amante par l'homme. La rencontre des principes antagonistes mâle et femelle dilue la différence dans un faire transformateur, qui n'épargne aucun genre, ne culpabilise spécifiquement aucun genre mais montre qu’en réalité le désir et l'amour sont l'apanage de personne. La femme amoureuse est aussi victime de la déchéance morale, du fait même du plaisir qu'elle en tire et qui finit par l'entraîner et par l'enchaîner à l'homme idéalisé et désiré. On peut l'observer dans le poème « Diable exquis» (A. S. Bonono, 2002 : 34) :

\author{
Tu es ma passion infernale, \\ Toi, figure emblématique d'Asmodée \\ Toi qui me véhicules aux enfers \\ De la vie néantisée $[\ldots]$ \\ Toi mon Dieu, mon Diable.
}

On note évidemment la substitution de l'image judéo-chrétienne de la femme incarnant le démon à celle de l'homme-bourreau, de l'homme-dieu et de l'homme-diable, facteur d'élévation et facteur de descente voire de déchéance de la femme, la transportant dans l'extase du septième ciel et la ramenant dans les souffrances et les ténèbres de l'enfer. Cette double postulation de l'amour masculin re-proportionne le mythe de l'éternel féminin et permet à la parole de devenir la panacée des souffrances provoquées par le désir du mâle et le désir de la femelle. 
En somme, à travers l'inversion du sens du désir, Angeline Solange Bonono restructure un ordre psycho-affectif et moral patriarcal qui ruine habilement le mythe de l'éternel féminin en présentant la femme non pas seulement comme une destructive de l'homme dans la volupté mais aussi comme un être détruit par ce dernier lorsqu'elle se lance à sa recherche. $\mathrm{Ce}$ bouleversement de l'ordre patriarcal dans l'art ne se conçoit pas indépendamment du renoncement aux mythes et aux clichés qui organisent les rapports entre le féminin et le masculin. Finalement, la parole scripturale de la poétesse est une parole sociale, à la fois féminine et masculine, qui dit les égarements de l'âme à l'époque contemporaine. Cette écriture du désir de la soif - rend compte de la complexité de la parole androgyne de la poétesse et des choix langagiers dénués de préciosité qu'elle engage dans son recueil de poèmes. Selon Raymond Jean (1977 : 9), en effet,

Ce désir initial est sans doute l'une des plus sûres données qui permette de rendre compte de la jonction qui s'accomplit entre le vécu et l'écriture dans un acte littéraire, l'une articulant le " projet désirant » de l'autre, et résolve ainsi dialectiquement l'éternelle et tenace contradiction entre la chose dite et la forme dans laquelle elle est dite.

L'écriture d'Angeline Solange Bonono est une écriture de la rencontre du féminin et du masculin. Sans toutefois privilégier l'un ou l'autre de ces genres, sa parole scripturale évolue vers une neutralisation de leurs différences.

\section{Vers une écriture neutre}

On peut situer l'écriture d'Angeline Solange Bonono dans le contexte camerounais, secoué par des revendications diverses dont la brisure de l'opacité du patriarcat n'est pas la moindre. Cette revendication primordiale s'inspire du féminisme et ses variantes. On peut noter d'emblée que la poétesse privilégie un féminisme universaliste qui met en avant l'humain au détriment du sexe. Dans le poème " Je refuse " (A. S. Bonono, $2002: 23$ ), elle qualifie sa plume d'" androgyne ": " Je refuse et je crie à travers ma plume androgyne ". Dans cette isonomie du féminin et du masculin, où se neutralisent, à la manière de l'oxymore, deux principes antagonistes, on peut y lire le désir d'unir la voix féminine originelle à la voix masculine acquise dans l'art, pour parler de l'humain tout court. En effet, selon Nicole Berry (1987 : 200), 
La neutralité serait un libre jeu entre des identifications. Elle se situerait entre ces deux extrêmes : la fixité de l'identification projective et la versatilité narcissique. Elle supposerait l'affirmation virile et l'effacement du féminin.

On l'a vu, la parole scripturale de la poétesse oscille, en effet, entre une spécularité féminine et une altérité masculine, entre la volonté de puissance du moi poétique et la virilisation de la parole, le but recherché étant de restructurer un ordre patriarcal surfait dans l'imaginaire social. La complémentarité des principes féminin et masculin ne fait aucun doute dans son écriture. L'usage de la parole virile n'est donc pas un simple travestissement qui aboutit à une identité masculine caricaturale de la poétesse, aussi pitoyable que peut l'être un poète, qui s'effémine. La parole virile est l'expression d'une liberté désirée et rêvée. Si elle transgresse et subvertit une norme traditionnelle omettant d'intégrer les principes féminin et masculin dans toute écriture de soi, elle reste, malgré tout, la quête ultime d'une audience sociale non différentialiste. Car, en réalité, qui n'est touché de près ou de loin par les forces du désir? Qui n'est effleuré par le pouvoir destructeur de la passion? Au total, la poétesse reconsidère l'antagonisme féminin et masculin en montrant que ces deux genres n'échappent pas au destin du commun des mortels. Elle transite par la thématique de l'amour et de la mort pour associer le genre humain dans un même destin. Dans le poème "Demain est un autre jour " (A. S. Bonono, 2002 : 51), on peut lire :

Quand aimer l'homme quand aimer la femme

Dans son lit devient nausée

Quand Thanatos devient Eros

Quand manger devient martyr

Quand haïr devient vade mecum

Il faut se dire malgré tout

Que ça ira mieux demain.

On peut voir la même tendance à philosopher devant la mort dans le poème "Ainsi va la mort " (A. S. Bonono, $2002: 38$ ) :

Nous avons mordu à tort ou à

Raison des morsures mortelles

Surtout! Ne pleurez pas! 
Nous ne valions rien

Et puis il faut être philosophe et se dire :

"On ne sort jamais vivant de la vie".

En somme, l'amour, la mort, le désir, etc., ne sont ni l'apanage du féminin, ni celui du masculin, mais celui du genre humain tout court. La parole scripturale de la poétesse, parole virile dans laquelle elle efface son origine féminine, répond certainement à un besoin d'affirmation dans une société patriarcale, où la voix masculine fait autorité. En effet, où la parole sociale lui est accordée de façon mitigée dans la réalité, la poétesse se saisit d'elle librement dans l'imaginaire. Le texte poétique devient le lieu de l'auto-déclamation et l'auto-théorisation d'une frustration sociale. Roland Barthes (1953:14) en mentionne l'influence sur la parole scripturale. Selon lui,

L'écriture est un acte de solidarité historique [...] Elle est le rapport entre la création et la société, elle est le langage littéraire transformé par sa destination sociale, elle est la forme saisie dans son intention humaine et liée ainsi aux grandes crises de l'Histoire.

Aujourd'hui, on ne peut méconnaître la légitimité du besoin d'épanouissement qui anime la femme et qu'elle expérimente sur les plans vestimentaire, psycho-affectif, intellectuel et professionnel, entre autres. La parole virile d'Angeline Solange Bonono, dans Soif Azur, est la variante littéraire de cette quête de la liberté de la femme qu'elle rapporte cependant à l'échelle masculine, prise comme mesure de légitimité dans les sociétés patriarcales. Sa poésie anticipe sur l'amélioration de la condition féminine par une restructuration de l'ordre patriarcal dénoncé. La virilisation de sa parole scripturale apparaît ainsi comme l'affirmation d'une liberté qu'elle atteint surtout dans l'art. Sa plume, véritable harmonisatrice de la différence entre le féminin et le masculin, inscrit son écriture dans un baroque résolument postmoderne exprimant les impasses d'un monde où visiblement la guerre des sexes commence à s'estomper.

\section{Bibliographie}

Barthes, Roland (1972), Le Degré zéro de l'écriture, "Qu'est-ce que l'écriture? ", Paris, Éditions du Seuil. 
Berry, Nicole (1978), Le Sentiment d'identité, " Le psychanalyste : masculin, féminin ou neutre? ", Begedis, Éditions universitaires, Coll. "Émergences".

Bonono, Angeline Solange (2002), Soif Azur, Yaoundé, Éditions de la Ronde.

Bourdieu, Pierre (1992), Les Règles de l'art. Genèse et structure du champ littéraire, Paris, Éditions du Seuil, Coll. "Points Essais".

Cros, Edmond (1998), Genèse socio-idéologique des formes, "L'écriture comme espace de l'autonomie ", Paris, Éditions du Centre d'études et de recherches sociocritiques, Coll. "Études sociocritiques".

Genette, Gérard (1976), Figures I, "Complexe de Narcisse ", Paris, Éditions du Seuil.

Goethe, Jean Wolfgang (1964), Faust, Traduction de Gérard de Nerval, Paris, Garnier Flammarion.

Jean, Raymond (1977), Lectures du désir : Nerval, Lautréamont, Apollinaire, Eluard, Paris, Éditions du Seuil, Coll. " Points".

Nietzsche, Friedrich (1993), Euvres, "Aurore : subtilité du sentiment de puissance ", Paris, Robert Laffont.

Starobinski, Jean (1964), La Relation critique, "Le sens de la critique ", Paris, Éditions Gallimard, Coll. "Le Chemin ».

Valette, Bernard (1990), Le Couple fatal, Paris, Éditions Bordas, Coll. «Les Thèmes littéraires ".

Yahi, Salem (1995), Sexualité et spiritualité, Paris, Éditions Françaises du Graal, Coll. "Les Valeurs de la vie». 